of soft scanning force microscope cantilevers. These cantilevers have oscillations perpendicular to the surface of the order of $10^{-10} \mathrm{~m}$, while a particle in an optical trap can fluctuate as much as several hundred nanometers in any direction. In this manner, the particle is able to scan over an area becoming a "natural 3-D mechanism".

Scientists from the European Molecular Biology Laboratory in Germany obtained 3-D images of an agar gel by means of this technique, as they describe in the November 26, 2001, issue of Applied Physics Letters. An agar gel is a transparent substrate used for biological cultures and chromatography. In this case, liquid agar was mixed with latex beads with a nominal diameter of $216 \pm 8 \mathrm{~nm}$. The beads were added at a concentration of $1 / 20 \mathrm{~mm}^{3}$. A photonic force microscope, which is a scanning force microscope based on optical tweezers, was used to conduct the experiments. It included a 1064-nm laser beam and a three-dimensional detector. This detector consists of a quadrant photodiode where the scattered laser light from the particle produces an interference pattern. The position of the optical trap is measured with $<1 \mathrm{~nm}$ error; the position of the center of the particle is measured with nanometer resolution.

A position histogram was calculated after recording the position of a particle for $0.8 \mathrm{~s}$. The thermally induced particle fluctuations allowed it to scan a volume of $300 \times$ $120 \times 120 \mathrm{~nm}^{3}$. It was also possible for the particle to scan areas around an object. When the optical trap was moved along the agar network in 80-nm steps, the variations on the position of the particle showed indications of the presence of the network filaments. The same effect was observed when the optical trap was moved along the $x$ and $y$-axes of the agar network in 80-nm steps and along the $\mathrm{z}$-axis in $160 \mathrm{~nm}$ steps. After scanning a volume of $600 \times 600 \times 300$ $\mathrm{nm}^{3}$, three filaments of the agar network were detected: two of them located inside this volume and one at the edge. The size of the particle affects the resolution which, in these experiments, is $\sim 20 \mathrm{~nm}$.

SIARI S. SOSA

\section{Micro-Optical Devices Dry Etched into Diamond}

Diamond has the widest optical transparency window and highest thermal conductivity of all known substances. Consequently, it is a very versatile material for use in optics. Diffractive optical elements (DOEs) are interesting for many applications, including the shaping of $\mathrm{CO}_{2}$ lasers and $\mathrm{Nd}$ :YAG lasers. As reported in the November 15, 2001, issue of Optics Letters, M. Karlsson, K. Hjort, and F. Nikolajeff fabricated continuousrelief blazed gratings and diffractive Fresnel lenses in diamond of optical quality at the Angstrom Laboratory in Sweden. The DOE pattern was first written in an electron-sensitive resist by direct-write electron-beam lithography, then transferred from the resist into the diamond by dry-etching in an inductively coupled plasma. The researchers used electronic-grade $\mathrm{O}_{2}$ and Ar gases for etching of the diamond substrate, and the surfaces were polished to a root-meansquare (rms) roughness of $<15 \mathrm{~nm}$

The transfer of the DOEs from the electron-beam resist into diamond was made in an inductively coupled plasma (ICP) etching system. ICP etching is a highdensity system with plasma-etch rates of $1900 \mathrm{~nm} / \mathrm{min}$ in resist and $190 \mathrm{~nm} / \mathrm{min}$ in diamond. The researchers reported that to their knowledge, this is the first time that dry etching has been used to transfer continuous structures into diamond. Both blazed gratings and diffractive Fresnel-type lenses were created. The transferred blazed grating was carefully inspected with both an optical microscope and with atomic-force microscopy (AFM) to measure the height of the structure, the surface morphology, and the accuracy of the transfer process. The blazed grating was found to have a period of $45 \mu \mathrm{m}$ and a grating depth of $3 \mu \mathrm{m}$. By using AFM data from the original structure, researchers simulated a diffraction efficiency of $71.5 \%$ for the e-beamexposed structure with a theoretical value of $100 \%$. After etching, the final diamond structure had a diffraction efficiency in the first order of $68.2 \%$, measured with a solid-state laser.

The group now intends to proceed with a more fundamental study of transferring micro-optical elements into diamond by using ICP etching. They also plan to fabricate diffractive and refractive elements for use with Nd:YAG high-power lasers. In this study, the researchers were unable to design optical elements for wavelengths larger than $\sim 700 \mathrm{~nm}$. However, they believe that replicating DOEs in photoresist might lead to optical elements for the infrared region, facilitating applications for 10.6- $\mu \mathrm{m} \mathrm{CO}_{2}$ lasers.

JENNIFER BURRIS

\section{Reduction in Roughness of Waveguides Leads to Ultralow Transmission Loss}

By reducing the sidewall roughness of $\mathrm{Si} / \mathrm{SiO}_{2}$ waveguides, researchers at the Massachusetts Institute of Technology and the University of Wisconsin-Madison have achieved ultralow transmission losses of $0.8 \mathrm{~dB} / \mathrm{cm}$ by using oxidation smoothing and anisotropic etching techniques. They believe this to be the smallest loss for a high-index-difference system reported to date.

Scattering losses in waveguides-as calculated by the commonly used method of Marcuse, Payne, and Lee-are proportional to the root-mean-square (rms) surface roughness. Typical rms roughness values for waveguides fabricated by conventional photolithography and reactive ion etching (RIE) techniques are about $10 \mathrm{~nm}$, which yield scattering losses of about $32 \mathrm{~dB} / \mathrm{cm}$. By following the photolithography and RIE steps with wet oxidation smoothing or anisotropic etching, the researchers were able to reduce the sidewall roughness to $2 \mathrm{~nm}$.

As reported in the December 1, 2001, issue of Optics Letters, K. Lee, D. Lim, and L. Kimerling at MIT, and J. Shin and F. Cerrina at Wisconsin first fabricated waveguides using standard methods. Starting with wafers consisting of a $0.2-\mu \mathrm{m}-$ thick layer of $\mathrm{Si}$ on 1- $\mu$ m-thick $\mathrm{SiO}_{2}$, they patterned the surface using UV photolithography, and etched it with $\mathrm{SF}_{6}$ gas. This produced straight waveguides with widths in the range of $0.5 \mu \mathrm{m}-8 \mu \mathrm{m}$. For the oxidation-smoothing sample, the Si layer of the starting wafer was $0.34 \mu \mathrm{m}$ thick. After photolithography and RIE, the waveguide core was subjected to wet oxidation by $\mathrm{H}_{2} \mathrm{O}$ and $\mathrm{O}_{2}$ at $1000^{\circ} \mathrm{C}$ for $43 \mathrm{~min}$ followed by an HF dip to remove the oxide.

The anisotropic-etching sample fabrication was more complicated. Again starting with a $0.34-\mu m$-thick Si layer on a 1- $\mu$ mthick $\mathrm{SiO}_{2}$ wafer, they oxidized the $\mathrm{Si}$ top layer to produce a 0.3 - $\mu$ m-thick $\mathrm{SiO}_{2}$ layer on a $0.2-\mu \mathrm{m}$-thick $\mathrm{Si}$ core, leaving the $1-\mu \mathrm{m}$ thick $\mathrm{Si}$ base layer intact. The top $\mathrm{SiO}_{2}$ layer was subject to photolithography and RIE to make a hard mask for the core of the waveguide, then the sample was immersed in a $25-\mathrm{wt} \%$ aqueous solution of $\mathrm{NH}_{4} \mathrm{OH}$ at $80^{\circ} \mathrm{C}$ for $1 \mathrm{~min}$ to etch away the $\mathrm{Si}$ from the non-waveguide area. The oxide hard mask was removed, leaving a Si waveguide core.

Atomic force microscopy revealed rms surface roughness values of $2 \mathrm{~nm}$ for both the oxidation-smoothed and the anisotropically etched samples, versus $10 \mathrm{~nm}$ for the conventionally fabricated waveguide. Transmission-loss measurements versus waveguide width were performed using cutback, IR capture of scattered light, and Fabry-Perot resonance measurements. The reduced transmission loss due to smoother surfaces in the oxidation- 